\section{Desastres naturais e seus custos nos estabelecimentos de saúde no Brasil no período de 2000 a 2015}

\section{Natural disasters and their costs for healthcare establishments in Brazil, 2000 to 2015}

\author{
Desastres naturales y sus costes para los \\ establecimientos de salud en Brasil, \\ período de 2000 a 2015
}

Carlos Machado de Freitas 1,2

Isadora Vida de Mefano e Silva 1,2

Diego Ricardo Xavier ${ }^{3}$

Eliane Lima e Silva 4

Christovam Barcellos 3 doi: 10.1590/0102-311X00133419

\section{Resumo}

Desastres naturais resultam em impactos na saúde das populações, danos aos estabelecimentos de saúde e, em situações extremas, colapso dos sistemas de saúde. Tendências nacionais e globais evidenciam o aumento da frequência dos desastres associados às mudanças climáticas. O objetivo deste artigo é analisar os impactos e custos econômicos dos desastres naturais sobre os estabelecimentos de saúde, identificando tipos mais frequentes e de maior custo, e distribuição no território nacional, tendo como base os dados registrados no Sistema Integrado de Informações sobre Desastres (S2ID) no período de 2000 a 2015. Foram sistematizados e analisados 15.950 registros, sendo que deste universo, em somente 29,4\% das ocorrências havia registros de custos, totalizando quase $R \$ 4$ bilhões. Os desastres climatológicos foram os mais recorrentes, mas não os responsáveis pelos custos mais expressivos. Na relação custos por evento, os desastres hidrológicos apresentaram custos 3,2 e 3,6 vezes maiores do que os meteorológicos e geológicos. Em relação ao custo total em milhões de reais nos estados, destacaram-se Pernambuco, Amazonas e Santa Catarina. Em relação ao custo por desastre em milhões de reais o destaque foi a Região Norte e em particular o Acre. Apesar das limitações deste estudo relacionadas à qualidade dos registros, os dados apresentados devem ser compreendidos como a ponta visível de um iceberg, pois os impactos e danos vão além dos econômicos, com impactos sobre a infraestrutura e recursos que servem de suporte aos serviços, comprometendo a capacidade de oferta exatamente quando a população mais necessita dos serviços de saúde.

Desastres Naturais; Instalações de Saúde; Custos e Análise de Custo; Mudança Climática

\author{
Correspondência \\ C. M. Freitas \\ Centro de Estudos e Pesquisas em Emergências e Desastres em \\ Saúde, Fundação Oswaldo Cruz. \\ Av. Brasil 4036, sala 916, Rio de Janeiro, RJ 21040-361, Brasil. \\ carlosmf@ensp.fiocruz.br \\ 1 Centro de Estudos e Pesquisas em Emergências e Desastres em \\ Saúde, Fundação Oswaldo Cruz, Rio de Janeiro, Brasil. \\ 2 Escola Nacional de Saúde Pública Sergio Arouca, Fundação \\ Oswaldo Cruz, Rio de Janeiro, Brasil. \\ 3 Instituto de Comunicação e Informação Científica e \\ Tecnológica em Saúde, Fundação Oswaldo Cruz, Rio de Janeiro, \\ Brasil. \\ 4 Departamento de Geografia, Universidade de Brasília, \\ Brasília, Brasil
}




\section{Introdução}

Em 2011, a Organização Mundial da Saúde (OMS) aprovou a resolução WHA64.10 1, que trata da necessidade de fortalecimento dos sistemas de saúde nacionais para a gestão de desastres e resiliência dos sistemas e serviços de saúde, de modo que não tenham sua capacidade de funcionamento comprometida nestes eventos. Foi precedida de uma série de resoluções relacionadas às ações do setor saúde em crises e desastres 2 , preparação e respostas às emergências 3 e mudanças climáticas e saúde 4 . Representam o reconhecimento da responsabilidade do setor saúde em relação às crises e desastres naturais, intensificados pelas mudanças climáticas, como também a necessidade de melhor preparação e resiliência para enfrentar estes desafios.

Nos âmbitos internacional e nacional foram ampliadas as preocupações em relação aos impactos das mudanças climáticas sobre a saúde, envolvendo as tendências do aumento da frequência e magnitude dos eventos climáticos extremos e desastres 5,6,7. No Brasil, cerca de $98 \%$ dos desastres de origem natural (hidrológicos, climatológicos e meteorológicos) ocorridos entre 1991 e 2010 estavam diretamente relacionados à variabilidade e mudanças climáticas e, mesmo os $2 \%$ restantes (geológicos) também apresentaram associação, pois a maioria envolveu movimentos de massa úmida provocados por chuvas contínuas ou intensas. Foram cerca de 32 mil desastres registrados com mais de 96 milhões de afetados que, além de resultarem em morbidade e mortalidade, geraram perdas e danos na infraestrutura, inclusive a de saúde 8.

A OMS vem continuamente alertando para a necessidade de preparação do setor saúde para enfrentar os desafios dos cenários que envolvem a variabilidade e as mudanças climáticas, com tendências na elevação da frequência e gravidade dos desastres de origem natural, como secas, inundações, deslizamentos de terra provocados por chuvas intensas, ciclones e furacões 9 . Esses eventos, além dos seus potenciais impactos na saúde das populações expostas, causam danos e prejuízos aos estabelecimentos de saúde e, em situações extremas, o colapso dos sistemas de saúde locais. Isso significa que exatamente quando os serviços de saúde são demandados a responder aos riscos adicionais provocados pelos desastres, os estabelecimentos de saúde podem ser afetados de modo direto, por meio de danos e destruições que podem impactar também os trabalhadores da saúde, ou mesmo indireto, com o comprometimento da infraestrutura básica, como suprimento de água e energia, entre outros. Em ambas as situações a capacidade de resposta do setor saúde é comprometida 10.

No Brasil, o Plano Nacional de Adaptação à Mudança do Clima (PNA) 7 (p. 207) estabelece para o setor saúde a necessidade de medidas de adaptação do sistema de saúde, tendo como diretriz o “... fomento e promoção de estabelecimentos de saúde sustentáveis, resilientes e seguros no âmbito do SUS e da Saúde Suplementar para a continuidade dos serviços de saúde nos cenários de desastres, insegurança hídrica e energética...”. Apesar de existirem trabalhos e relatórios sobre danos a instalações de saúde decorrentes de alguns desastres específicos, este estudo consiste no primeiro levantamento no nível nacional e durante um longo período de tempo sobre os custos causados ao setor saúde por desastres naturais, o que permite avaliar a vulnerabilidade dos serviços de saúde e sua capacidade de reposta em condições de emergência.

O objetivo deste artigo é analisar os impactos e custos econômicos dos desastres naturais sobre os estabelecimentos de saúde, identificando quais tipos são mais frequentes e de maior custo, e sua distribuição nas regiões e Unidades da Federação (UF) do país, tendo como base os dados de desastres naturais ocorridos no país no período de 2000 a 2015, registrados no Sistema Integrado de Informações sobre Desastres (S2ID), da Secretaria Nacional de Proteção e Defesa Civil (SEDEC).

\section{Método}

No Brasil, até a publicação da Instrução Normativa no 1, de 24 de agosto de 2012, do Ministério da Integração Nacional 11, o registro oficial de um desastre poderia ocorrer pela emissão, por parte dos municípios afetados, de três formulários distintos, não obrigatoriamente dependentes: uma Notificação Preliminar de Desastre (NOPRED), uma Avaliação de Danos (AVADAN) ou um Decreto de Prefeitura. Após a publicação dessa Instrução Normativa, o NOPRED e o AVADAN foram substituídos por um único documento, o Formulário de Informações sobre Desastres (FIDE) 12. 
Para o presente estudo, foi realizado um levantamento dos dados de desastres naturais ocorridos no Brasil no período de 2000 a 2015, resultando num total de 15.950 formulários de registros. Os eventos foram organizados tendo como referência a Classificação e Codificação Brasileira de Desastres (COBRADE). Essa nova classificação foi estabelecida pela mesma Instrução Normativa no 1, que em seu artigo 7o esclarece que o Brasil passou a adotar a classificação e simbologia adotadas pelo Banco de Dados Internacional de Desastres (EM-DAT), do Centro para Pesquisa sobre Epidemiologia de Desastres (CRED), reconhecidas como oficiais por órgãos das Nações Unidas como a Estratégia Internacional de Redução de Desastres (EIRD/UNDRR) e a OMS 11.

Os formulários de registros de desastres em arquivos no formato PDF foram obtidos no S2ID, da SEDEC, onde estão disponíveis de forma pública. Esse sistema registra os desastres informados pelos municípios ao órgão estadual de Defesa Civil ou à SEDEC. As principais variáveis desses formulários foram redigitadas e passaram a compor um banco de dados sobre desastres, disponibilizados pelo Observatório de Clima e Saúde 13.

No processo de desenvolvimento desta pesquisa, que nos levou aos resultados que apresentaremos no próximo tópico, foram utilizados os dados (custos em $\mathrm{R} \$$ ) da tabela de danos materiais contidos nos formulários de registros dos eventos selecionados. Esses dados, como mencionamos antes, foram coletados segundo diferentes formulários, preenchidos pela autoridade municipal.

O NOPRED, apesar de ter um campo para os danos materiais em seu item número 6, não apresenta dados específicos sobre os danos materiais em saúde. Esse tipo de formulário representa 2,8\% dos formulários de registros levantados.

Os formulários da AVADAN contêm os dados dos danos materiais e edificações em seu item número 7, do qual foram extraídas as informações específicas referentes aos danos em estabelecimentos de saúde, públicos e particulares. Esse tipo de formulário representa $80,5 \%$ de todos os registros.

Os formulários FIDE, que representam 16,7\% da totalidade dos registros, têm os dados referentes aos danos materiais no seu item 6.2. Nesse item, entretanto, não há um campo específico para o preenchimento de informações de danos em estabelecimentos particulares de saúde, o que justifica que, para o presente trabalho, tampouco se tenha discriminado os prejuízos em estabelecimentos públicos ou particulares.

Dos formulários que tinham campo específico para registros dos danos materiais em instalações de saúde (FIDE e AVADAN), 67,5\% não registravam informações sobre danos aos estabelecimentos de saúde e seus custos econômicos ( $\mathrm{R} \$)$, não sendo possível identificar se o campo vazio correspondia à ausência de custos ou à ausência de informação. Na interpretação dos autores, os registros que indicavam a ausência de custos em estabelecimentos de saúde tiveram, nesse campo específico, o valor de R\$ 0,00 preenchido pelos órgãos municipais responsáveis pelo registro.

Somando-se aos registros com campos vazios, aqueles que não têm campos específicos de danos materiais em saúde, e os NOPRED, os resultados referentes aos custos dos desastres em estabelecimentos de saúde são próximos de $29,4 \%$ da totalidade dos registros.

Para calcular o universo dos registros que compuseram os resultados referentes aos custos (em R\$) em estabelecimentos de saúde, foram excluídos: (1) os NOPRED, pois estes já não contam com este tipo de informação em seu formulário; (2) as AVADAN com os campos de danos materiais em saúde, pública e particular, vazios; (3) as AVADAN sem o campo de danos materiais em saúde; (4) os FIDE com o campo de danos materiais em saúde pública vazio. Excluindo esses formulários mencionados, os custos totais foram calculados somando-se os valores registrados no item referente aos danos materiais em edificações de estruturas públicas e particulares de saúde (no FIDE consta no item 6.2, na AVADAN consta no item 7) do universo dos formulários que compuseram de fato o total dos custos estimados no estudo.

Adotava-se no país a Codificação de Desastres, Ameaças e Riscos (CODAR), que está em dissonância com a classificação das principais bases de dados internacionais sobre desastres naturais (SIGMA, NatCatSERVICE e EM-DAT) 14. Em agosto de 2012, o Brasil passou a adotar para a classificação dos desastres a COBRADE como classificação oficial. Neste estudo adotamos para todo o período a COBRADE, que está em sintonia com as bases de dados internacionais e com os órgãos das Nações Unidas (EIRD e OMS).

Para uma melhor adaptação à realidade brasileira, enquadramos todas as ocorrências associadas a movimentos de massa, como desastres geológicos, de acordo com a classificação adotada pela 
COBRADE. Apesar de no Brasil a maioria dos movimentos de massa ser composto por corrida de massa úmida, estando assim associados à ocorrência de chuvas e, portanto, serem considerados desastres hidrológicos pela classificação do EM-DAT, a COBRADE os classifica como geológicos.

Dessa forma, os desastres foram associados a uma das quatro classificações de tipos:

(1) Geológicos ou geofísicos: envolvem os processos erosivos, de movimentação de massa (incluídos os resultantes de movimentação de massa úmida e deslizamentos derivados de processos geológicos ou fenômenos geofísicos).

(2) Meteorológicos: envolvem os processos que resultam em fenômenos como chuvas intensas, raios, ciclones tropicais e extratropicais, tornados e vendavais, chuvas de granizo, geadas e ondas de frio e de calor.

(3) Hidrológicos: envolvem os processos que resultam em alagamentos, enchentes, inundações graduais e bruscas.

(4) Climatológicos: envolvem os processos relacionados à estiagem e seca, queimadas e incêndios florestais.

As informações dos registros usadas para gerar resultados e discussão estão relacionadas à classificação do tipo de desastre, ao número de ocorrências (resultante do número de registros por classificação de tipo de desastre) no período de 2000 a 2015, à UF e região de ocorrência, e aos seus danos materiais, especificamente aqueles relacionados aos custos $(\mathrm{R} \$)$ em estabelecimentos de saúde dos municípios afetados.

\section{Resultados}

Observa-se que, considerando a classificação de desastres "naturais" ocorridos no Brasil no período de 2000 a 2015, predominaram os climatológicos com 56,2\%, seguidos dos hidrológicos com 34,9\%, meteorológicos com $8 \%$ e, por fim, os geológicos com $0,9 \%$ do total do número de ocorrências. Os dados apontam grande variabilidade, com tendência de crescimento do número de ocorrências nesse mesmo período (Figura 1).

\section{Figura 1}

Evolução temporal por classificação de tipo de desastres e por custos. Brasil, 2000-2015.

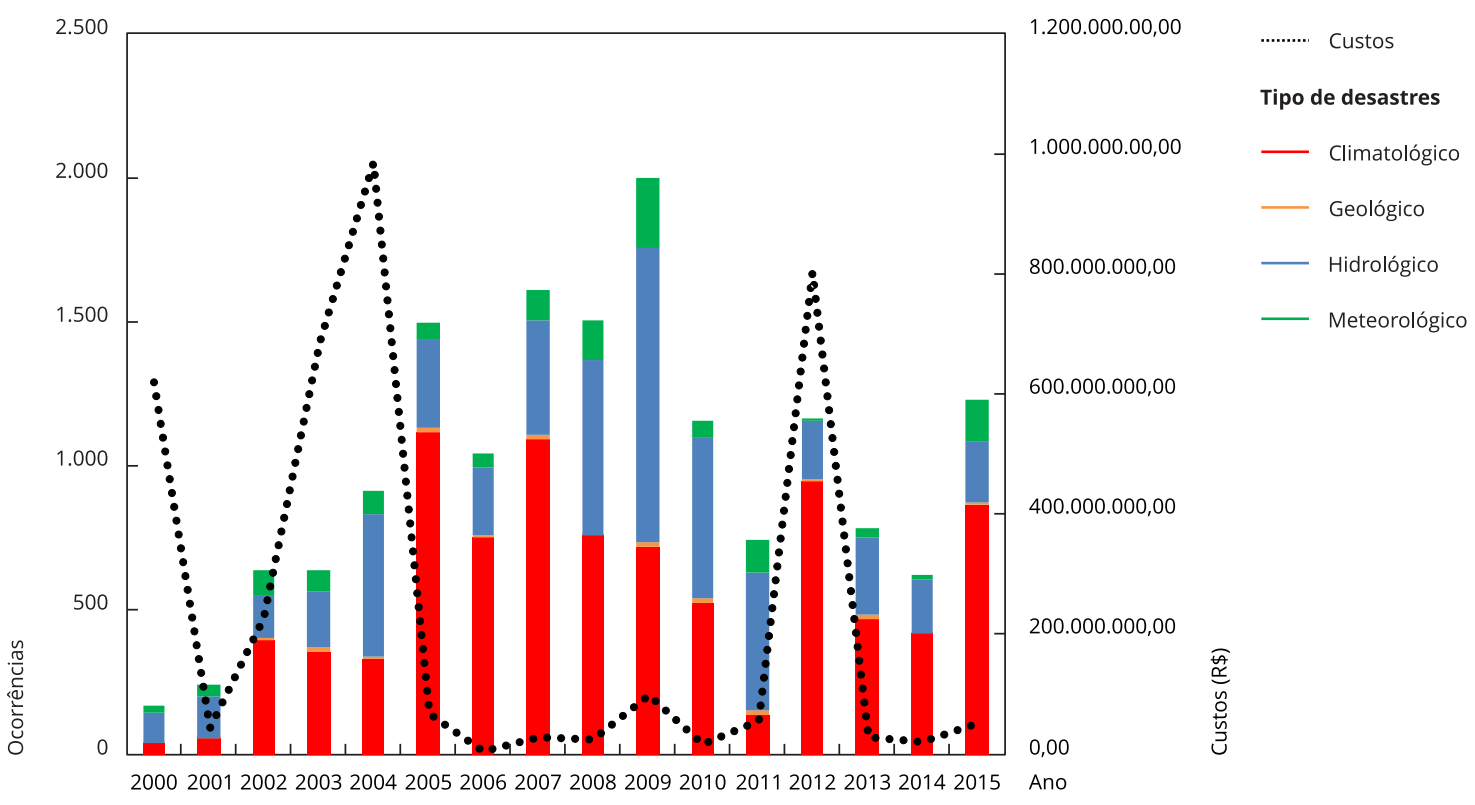


De acordo com o levantamento deste estudo, os custos econômicos totais dos desastres em estabelecimentos de saúde, no Brasil, foram de quase R 4 bilhões (Tabela 1). Entretanto, como é possível perceber na Figura 1, os custos mais elevados nem sempre estão associados a uma maior frequência de eventos, apresentando os maiores valores nos anos de 2000, 2004 e 2012.

A Tabela 1 apresenta a relação dos custos totais por número de ocorrências ao trazer, no seu quantitativo de ocorrências, tanto os registros totais quanto aqueles que de fato compuseram os resultados relativos aos custos, presentes em apenas $29,4 \%$ dos registros.

\section{Tabela 1}

Número de ocorrências e custos, utilizando-se tanto o quantitativo total de registros (apenas para dados relativos ao número de ocorrências) quanto apenas aqueles que de fato compuseram os resultados relativos aos custos. Brasil, 2000-2015.

\begin{tabular}{|c|c|c|c|c|c|c|}
\hline \multirow[t]{2}{*}{$\begin{array}{l}\text { Classificação de } \\
\text { tipo de desastre }\end{array}$} & \multirow[t]{2}{*}{ Tipo de desastre } & \multicolumn{2}{|c|}{$\begin{array}{l}\text { Ocorrências por } \\
\text { tipo de desastre }\end{array}$} & \multicolumn{2}{|c|}{$\begin{array}{l}\text { Ocorrência por } \\
\text { classificação de } \\
\text { tipo de desastre }\end{array}$} & \multirow[t]{2}{*}{$\begin{array}{c}\text { Custo por } \\
\text { classificação e tipo } \\
\text { de desastre }\end{array}$} \\
\hline & & Total & $\begin{array}{l}\text { Registros com } \\
\text { informações } \\
\text { sobre os custos }\end{array}$ & Total & $\begin{array}{l}\text { Registros com } \\
\text { informações } \\
\text { sobre os custos } \\
\text { [n (\%)] }\end{array}$ & \\
\hline \multirow[t]{5}{*}{ Climatológico } & Estiagens & 7.518 & 1.774 & 8.960 & $2.251(25,1)$ & $\mathrm{R} \$ 12.930 .389,00$ \\
\hline & Incêndios florestais & 7 & 1 & & & \\
\hline & Incêndios urbanos ou rurais & 1 & 0 & & & \\
\hline & Ondas de frio intenso & 2 & 0 & & & \\
\hline & Secas & 1.432 & 476 & & & \\
\hline \multirow[t]{5}{*}{ Geológico } & Boçorocas & 1 & 1 & 147 & $55(37,4)$ & $\mathrm{R} \$ 29.286 .605,00$ \\
\hline & Corridas de massa & 2 & 0 & & & \\
\hline & Deslizamentos de solo e/ou rocha & 15 & 15 & & & \\
\hline & Escorregamentos ou deslizamentos & 128 & 39 & & & \\
\hline & Rastejos & 1 & 0 & & & \\
\hline \multirow[t]{7}{*}{ Hidrológico } & Alagamentos & 207 & 126 & 5.568 & $1.751(31,4)$ & $\mathrm{R} \$ 3.337 .524 .644,71$ \\
\hline & Enchentes ou inundações graduais & 1.699 & 311 & & & \\
\hline & Enxurradas & 258 & 255 & & & \\
\hline & Enxurradas ou inundações bruscas & 3.130 & 788 & & & \\
\hline & Erosão marinha & 1 & 0 & & & \\
\hline & Inundações & 272 & 271 & & & \\
\hline & $\begin{array}{l}\text { Inundações litorâneas provocadas pela } \\
\text { brusca invasão do mar }\end{array}$ & 1 & 0 & & & \\
\hline \multirow[t]{9}{*}{ Meteorológico } & Chuvas intensas & 58 & 58 & 1.275 & $628(49,3)$ & $R \$ 372.830 .942,25$ \\
\hline & Geadas & 21 & 3 & & & \\
\hline & Granizos & 498 & 281 & & & \\
\hline & Tornados & 5 & 5 & & & \\
\hline & Tornados e trombas d'águas & 12 & 3 & & & \\
\hline & Vendavais & 34 & 32 & & & \\
\hline & $\begin{array}{l}\text { Vendavais extremamente intensos, furacões, } \\
\text { tufões ou ciclones tropicais }\end{array}$ & 16 & 13 & & & \\
\hline & $\begin{array}{l}\text { Vendavais muito intensos ou ciclones } \\
\text { extratropicais }\end{array}$ & 80 & 29 & & & \\
\hline & Vendavais ou tempestades & 551 & 204 & & & \\
\hline Total geral & & & & 15.950 & $4.685(29,3)$ & $R \$ 3.752 .572 .580,96$ \\
\hline
\end{tabular}


Observa-se que embora os desastres meteorológicos tenham apresentado o maior percentual de eventos com informações sobre custos (49,3\%), responderam por apenas 9,9\% dos custos totais. Por sua vez, os desastres hidrológicos embora tenham apresentado menos de um terço $(31,4 \%)$ dos eventos com informações sobre custos, responderam por 88,5\% dos custos totais. Os desastres geológicos, nos quais estão incluídos deslizamentos e escorregamentos, foram responsáveis por $0,8 \%$ dos custos totais. Por fim, os climatológicos foram os desastres com o menor percentual de registros sobre os custos, como também pelo menor percentual de custos totais, respondendo por apenas $0,3 \%$ dos mesmos.

A Figura 2 apresenta a distribuição dos custos aos estabelecimentos de saúde, segundo a classificação do tipo de desastre, utilizando-se apenas os registros que de fato compuseram os resultados relativos aos custos. Apesar dos desastres classificados como climatológicos serem os mais recorrentes no Brasil, eles não são os responsáveis pelos custos mais expressivos por desastres. A relação dos custos por eventos constata que os desastres hidrológicos são os mais dispendiosos e com o maior custo, sendo 3,2 e 3,6 vezes maiores do que os custos para os meteorológicos e geológicos, respectivamente. Os desastres climatológicos, com grande contribuição dos eventos de seca, chegam a ter custos 93 vezes menores do que os geológicos, que ocupam a terceira posição nos custos por desastres. Ao mesmo tempo, os desastres geológicos, que no quantitativo de ocorrências são quase inexpressivos, passam a chamar atenção quando considerados os custos por desastres.

A Tabela 2 permite uma análise do total de eventos por UF e regiões, bem como custos por desastre para ambas. As UF com o maior número de eventos e que tiveram pelo menos mil registros, foram, em ordem decrescente, Rio Grande do Sul, Minas Gerais, Santa Catarina, Bahia, Paraíba, Pernambuco e Ceará. Não por acaso, as regiões que concentram o maior número de desastres foram as dessas UF, com Nordeste, Sul e Sudeste registrando 7.288, 4.653 e 2.911 eventos, respectivamente. Em relação ao custo total em milhões de reais, Pernambuco registra mais de 900; Amazonas e Santa Catarina entre 400 e 700; Acre e Minas Gerais na faixa dos 300; Pará, Tocantins, Bahia e Rio de Janeiro na faixa dos 100. As UF com o maior custo por desastre em milhões de reais foram Acre com 15,7 e Tocantins com 2,9. Embora a Região Norte tenha registrado 719 eventos, que é menos de um décimo do total de desastres da Região Nordeste, o custo total dos desastres para cada uma destas regiões foi bastante próximo, ultrapassando um bilhão de reais. Esses custos mais altos na Região Norte, combinados com o segundo menor número de eventos, contribuíram para que esta região apresente o maior custo por evento, que foi de $\mathrm{R} \$ 1.527 .644,49$, sendo de 7 a 9 vezes maior que o custo por evento nas regiões Nordeste, Sudeste e Sul.

\section{Figura 2}

Custo por ocorrência por classificação de desastre. Brasil, 2000-2015.

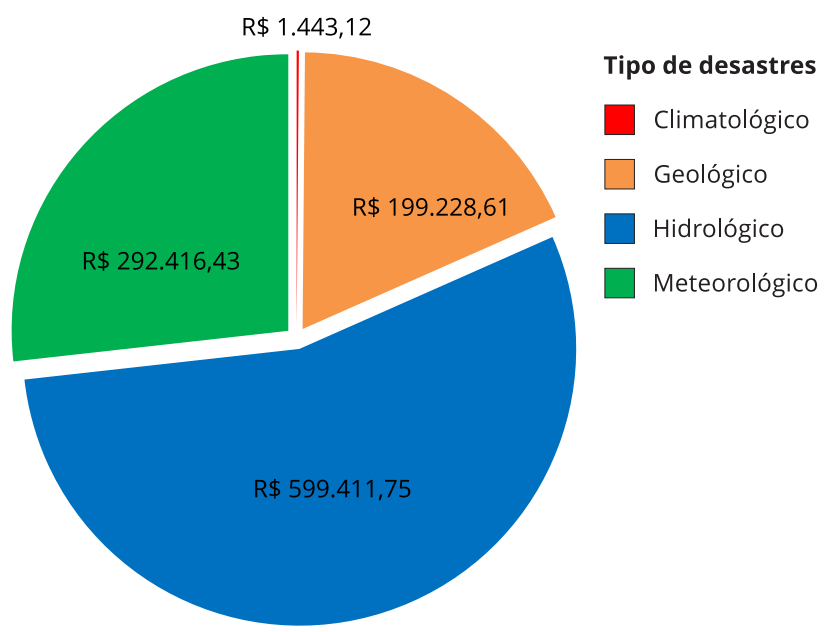


Tabela 2

Total de registros, custos e custos por evento por Unidades da Federação (UF). Brasil, 2000-2015.

\begin{tabular}{|c|c|c|c|}
\hline Região/UF & Total de registros por UF & Custo total (R\$) & Custo por evento por UF (R\$) \\
\hline \multicolumn{4}{|l|}{ Norte } \\
\hline Rondônia & 23 & $1.136 .250,00$ & $49.402,17$ \\
\hline Acre & 22 & $346.198 .550,00$ & $15.736 .297,73$ \\
\hline Amazonas & 327 & $474.277 .067,68$ & $1.450 .388,59$ \\
\hline Roraima & 44 & - & - \\
\hline Pará & 247 & $123.909 .118,04$ & $501.656,35$ \\
\hline Amapá & 4 & $28.400,00$ & $7.100,00$ \\
\hline Tocantins & 52 & $152.827 .000,00$ & $2.938 .980,77$ \\
\hline \multicolumn{4}{|l|}{ Nordeste } \\
\hline Maranhão & 272 & $15.563 .000,00$ & $57.216,91$ \\
\hline Piauí & 959 & $417.900,00$ & 435,77 \\
\hline Ceará & 1.356 & $14.058 .649,00$ & $10.367,74$ \\
\hline Rio Grande do Norte & 618 & $45.961 .490,00$ & $74.371,34$ \\
\hline Paraíba & 1.003 & $356.490,00$ & 355,42 \\
\hline Pernambuco & 1.029 & $983.865 .050,00$ & $956.137,07$ \\
\hline Alagoas & 299 & $790.000,00$ & $2.642,14$ \\
\hline Sergipe & 162 & $311.000,00$ & $1.919,75$ \\
\hline Bahia & 1.590 & $137.563 .375,00$ & $86.517,85$ \\
\hline \multicolumn{4}{|l|}{ Sudeste } \\
\hline Minas Gerais & 1.858 & $361.977 .790,00$ & $194.821,20$ \\
\hline Espírito Santo & 483 & $28.490 .281,96$ & $58.986,09$ \\
\hline Rio de Janeiro & 154 & $189.349 .693,00$ & $1.229 .543,46$ \\
\hline São Paulo & 416 & $70.174 .550,00$ & $168.688,82$ \\
\hline \multicolumn{4}{|l|}{ Sul } \\
\hline Paraná & 952 & $55.520 .497,61$ & $58.319,85$ \\
\hline Santa Catarina & 1.761 & $658.238 .681,22$ & $373.786,87$ \\
\hline Rio Grande do Sul & 1.940 & $88.230 .451,00$ & $6.209,44$ \\
\hline \multicolumn{4}{|l|}{ Centro-oeste } \\
\hline Mato Grosso do Sul & 138 & $377.010,00$ & $2.731,96$ \\
\hline Mato Grosso & 180 & $2.950 .286,45$ & $16.390,48$ \\
\hline Goiás & 61 & - & - \\
\hline Distrito Federal & 0 & - & - \\
\hline
\end{tabular}

\section{Discussão}

Este estudo buscou dimensionar o impacto econômico dos desastres no sistema de saúde, contabilizando custos decorrentes de avarias ou mesmo destruição dos estabelecimentos de saúde. Os dados apontam que quase R 44 bilhões foram perdidos somente no período de análise e considerando apenas as informações registradas pela defesa civil. É importante destacar que não estão contabilizados os custos do processo de reconstrução e aquisição de equipamentos.

Observa-se que, considerando-se a classificação por tipos de desastres naturais ocorridos no Brasil, os dados de ocorrência apresentam números similares aos de outros estudos publicados sobre desastres e saúde no país 8,13 .

Tomando-se como referência a análise contida em um documento da Confederação Nacional dos Municípios (CNM) sobre desastres 15, constatamos que no período de 2012 a 2016 os prejuízos econômicos causados pelos desastres foram de $\mathrm{R} \$ 205$ bilhões, representando uma média de $\mathrm{R} \$ 41,1$ bilhões por ano. Minervino \& Duarte 16 realizaram um estudo sobre os danos materiais por desastres hidrológicos ocorridos no Brasil no período de 2010 a 2014, utilizando também dados do S2ID, 
identificando um prejuízo de 1,2 bilhão em 5 anos, o que resultou em um média de 240 milhões por ano. Em nosso estudo, abrangendo um período maior e todos os tipos de desastres naturais, pudemos constatar na Tabela 1 que os custos econômicos totais dos desastres em estabelecimentos de saúde foram de quase $\mathrm{R} \$ 4$ bilhões em 16 anos, o que representa uma média de 250 milhões por ano. Apenas para efeito comparativo, mesmo considerando períodos distintos, a média dos danos materiais dos estabelecimentos de saúde corresponde a $0,6 \%$ dos prejuízos médios anuais com desastres, quando comparada com o estudo da CNM 15.

Os dados sobre prejuízos econômicos são importantes para trazer à tona o problema dos danos aos estabelecimentos de saúde em desastres. Ao mesmo tempo podem revelar diferentes métodos de estimativas que devem ser aperfeiçoados e tornados mais transparentes, pois podem apontar tanto subestimação como superestimação de custos, ambas as situações relacionadas à qualidade dos registros, envolvendo desde ausência de padronização até a participação mais direta do próprio setor saúde na produção dos mesmos. Para melhor discussão ilustraremos com situações envolvendo desastres climatológicos, hidrológicos e geológicos.

Os desastres climatológicos apresentaram na Figura 2 custo por evento 416 vezes menor que os hidrológicos. A seca é um desastre extensivo e tem como característica a baixa severidade de perdas e danos imediatos, alta frequência de eventos e seu prolongamento no tempo, podendo durar até 6 anos no Brasil 17. O estudo da CNM 15 demonstra que no período 2012 a 2015 os prejuízos causados por desastres no país foram da ordem de R $\$ 173,5$ bilhões, sendo que deste total cerca de 64,2 bilhões (37\%) corresponderam aos prejuízos provocados pelas secas, que atingiram principalmente as UF da Região Nordeste. Dentro desses custos estão os relacionados ao comprometimento do abastecimento de água, que somaram mais de R\$13,8 bilhões em prejuízos. Esses custos do abastecimento de água atingem também os estabelecimentos de saúde que, assim como a população, necessitam de carros pipa para garantir o seu funcionamento. Não encontramos estudos que dimensionem esses custos para o setor saúde, resultando na subestimação ou mesmo ocultamento do problema.

Para os desastres hidrológicos, a Tabela 2 aponta que a Região Norte concentra o segundo maior custo total dos desastres, contribuindo para isto duas UF (Acre e Amazonas), que têm como eventos predominantes as inundações graduais, que representam $95 \%$ e $79 \%$ do total de eventos, respectivamente. Porém, quando comparamos os custos por desastre há uma grande disparidade, com o Acre que teve 15 vezes menos registros de desastres do que o Amazonas, apresentando um custo por evento cerca de 11 maior. A comparação entre as duas UF, bem como os dados gerais apresentados, aponta para uma possível superestimação dos custos nesse caso específico, o que pode estar ocorrendo em outras situações.

Os desastres geológicos são caracterizados como intensivos, apresentando baixa frequência, geograficamente concentrados e com grande potencial de perdas, danos e mortalidade. Enquanto os dados globais apontam para uma média de 6 vezes mais óbitos quando inundações são combinadas com deslizamentos, no Brasil estudos indicam que este número chega a ser quase 13 vezes mais 8 . Esses dados podem indicar também um maior potencial de danos aos estabelecimentos de saúde, muitas vezes localizados em encostas ou próximos a elas.

Nas chuvas fortes que resultaram em múltiplos deslizamentos de terra e enxurradas na Região Serrana do Rio de Janeiro, em janeiro de 2011, houve o maior desastre em termos de vítimas fatais no país e uma série de danos e destruição dos estabelecimentos de saúde 18. No sistema S2ID não foram encontrados registros contendo estimativa ou avaliação de danos desse evento, apenas uma portaria sobre a situação de emergência e calamidade pública. Entretanto, um relatório do Banco Mundial 19 sobre a avaliação das perdas e danos provocados por esse desastre estimou que os custos para o setor saúde foram de 11,2 milhões, correspondendo a $0,2 \%$ dos custos totais do mesmo, que foram de 4,7 bilhões.

Nesse desastre, um levantamento realizado pelo Ministério da Saúde em alguns dos municípios atingidos (Bom Jardim, Nova Friburgo, São José do Vale do Rio Preto e Sumidouro) constatou que de 43 estabelecimentos de saúde, $81 \%$ estavam localizados em áreas de risco ${ }^{18}$. Em Nova Friburgo, Silva 20 demonstrou que 7 anos após o desastre de 2011 os estabelecimentos de saúde permaneceram nas mesmas áreas de risco, sem que tenham sido tomadas medidas estruturais para reduzir a vulnerabilidade destes estabelecimentos, de seus profissionais e de seus usuários. Segundo o levantamento desse estudo, 79,2 \% dos estabelecimentos de saúde do município estão em áreas de risco de inundação e/ ou deslizamentos. 
Apesar das limitações deste trabalho relacionadas à qualidade dos registros e estimativas de custos, indicando a existência de sub-registros, subestimação ou superestimação de custos, os dados apresentados devem ser compreendidos como a ponta visível de um iceberg, pois os impactos e danos vão além dos financeiros. Os estudos apontam que os desastres têm impactos sobre os trabalhadores da saúde, bem como sobre a infraestrutura e os recursos que servem de suporte aos serviços, comprometendo a capacidade de oferta exatamente quando a população mais necessita dos serviços de saúde.

Logo após o Furacão Katrina o número de médicos de Nova Orleans (Estados Unidos) passou de 4.486 para 1.200 (redução de 73,3\%) 21. E mesmo em situações de desastres em que estabelecimentos de saúde não são atingidos, mas a região ou município foi afetado, os serviços de saúde podem ter o seu funcionamento comprometido por impactos sobre as equipes de saúde, com profissionais atingidos por óbitos e adoecimentos dos mesmos ou de familiares, bem como perdas de suas moradias ou outros bens 22. Para além dos impactos na infraestrutura, é importante observar que alguns profissionais de saúde deixam a comunidade antes ou depois de um desastre, pelo menos temporariamente, comprometendo o pleno funcionamento dos serviços e a capacidade de resposta do setor saúde 23 .

Um estudo realizado em países europeus apontou que os estabelecimentos de saúde inundados tiveram como consequências interrupção dos atendimentos e atividades de cuidados, evacuação de pacientes, interrupções no fornecimento de energia e água, bem como dos serviços de ambulância, entre outros 24. Carvalho 25, comparando o total de atendimentos da semana anterior ao desastre de 2011 com o da semana posterior ao mesmo, no principal hospital público municipal de Nova Friburgo, demonstrou que houve uma queda no total de atendimentos de 1.800 por semana para 526 (queda de 71\%). Uma breve análise das informações do Sistema de Internação Hospitalar do Sistema Único de Saúde (SIH/SUS) registrou durante as enchentes do Vale do Itajaí (Santa Catarina) em 2008 que o número de internações foi reduzido de 4.100 para cerca de 2.900 (queda de 70,7\%), sendo notável a perda de 242 internações devido a causas ligadas à gravidez, parto e puerpério no mês de dezembro de 2008.

Ao mesmo tempo, os impactos sobre a infraestrutura, como os relacionados ao acesso à água $\mathrm{e}$ saneamento, não só comprometem o funcionamento dos estabelecimentos de saúde e atendimento e cuidados para as doenças preexistentes na população, mas também provocam novos problemas de saúde 26.

Pessoas com doenças crônicas (renais, cardiovasculares, diabetes, dentre outras) podem ter seus quadros clínicos agravados em situações de desastres. Em áreas atingidas pelos Furacões Katrina e Rita, de $25 \%$ a $40 \%$ dos habitantes possuíam alguma doença crônica e foi observado um aumento de atendimentos 10 dias após o início do desastre 27. Nas enchentes no Vale do Itajaí, em 2008, o padrão do número de internações por acidente vascular cerebral (AVC) elevou, chegando a duplicar, mantendo-se alto ao longo dos seis meses subsequentes, o que, segundo o estudo, poderia estar relacionado ao colapso das ações na atenção primária à saúde e prevenção de doenças 28 .

Para as pessoas que possuem condições crônicas de saúde e um acesso precário durante os períodos de "normalidade" cotidiana, as situações de "anormalidade" que caracterizam um desastre podem exacerbar as mesmas pela redução ou interrupção do funcionamento dos serviços nos estabelecimentos de saúde 23. Com isso, as disparidades que existem no acesso à saúde antes de um desastre são acentuadas após, principalmente quando a estrutura de saúde é afetada, como ficou evidenciado no Katrina 26.

\section{Considerações finais}

Os dados apresentados e discutidos neste artigo demonstram que os registros da Defesa Civil revelam apenas uma parte dos impactos e custos diretos e indiretos que os desastres provocam nos estabelecimentos de saúde, os quais resultam no comprometimento do seu funcionamento durante e logo após os desastres. Porém, mesmo com essas limitações, é possível verificar a complexidade do tema, revelando que não existe uma relação direta entre a frequência dos tipos de desastres e seus custos, assim como entre as UF e regiões com maior número de eventos e custos, bem como os custos por eventos.

Utilizando a imagem de um iceberg, podemos considerar que para ir além da superfície que os registros atuais revelam sobre os custos dos desastres nos estabelecimentos de saúde é necessário ter 
em conta que sub-registros, subestimação ou superestimação integram a ausência de políticas públicas que permitam avaliações e estimativas sobre os impactos dos desastres em nossa sociedade por tipos e regiões.

Na Região Norte, as mesmas inundações graduais e amazônicas que atingiram o Acre resultaram em pelo menos 10 vezes mais eventos em outras UF como Amazonas e Pará, mas com custo por evento de 11 a 30 vezes menor, indicando possível superestimação dos custos. Ao mesmo tempo, esses eventos que integram o ciclo das águas na Região Norte acabam sendo naturalizados, de modo que as inundações que afetam as diversas construções nos municípios, incluindo os estabelecimentos de saúde, em muitos casos não são realizados os registros de impactos e custos, resultando também em subestimação. Na Região Nordeste, que concentra a maioria dos desastres por seca, o custo de $\mathrm{R} \$ 1.443,12$ por evento, cujo registro cobre em média 6 meses de duração, claramente aponta para uma subestimação e sub-registro dos custos, que inclui o abastecimento de água por carros pipa durante o período.

Estruturas do SUS, como secretarias municipais de saúde, estão presentes em todos os municípios brasileiros. Por outro lado, estruturas de Defesa Civil, como coordenações municipais, só existem em pouco mais da metade dos municípios (50,4\%), por meio de coordenação municipal 29. Sem políticas públicas que integrem ambos os setores de modo a estruturar registros comuns e compartilhados de desastres e saúde, e entre estes, os relacionados aos estabelecimentos de saúde, de modo a permitir avaliações e estimativas sobre os impactos destes eventos no setor saúde.

De qualquer modo, os dados apresentados neste artigo já apontam a importância de se adotar ações tendo como referência o PNA 7, que aponta para a necessidade de incorporar nas políticas de saúde tecnologias e infraestruturas resilientes aos eventos desastres, como: (1) unidades de saúde e códigos de construção que considerem avaliações de vulnerabilidades do próprio setor saúde, localização dos estabelecimentos, tipologias de desastres, riscos atuais e futuros relacionados ao clima; (2) processos e tecnologias que garantam suprimento de água e energia (incluindo a solar), telecomunicações e transporte de insumos de saúde, saneamento envolvendo drenagem e disposição de resíduos, de modo que os estabelecimentos de saúde se tornem preparados e adaptados para permanecerem funcionais durante eventos extremos 10 .

\section{Colaboradores}

C. M. Freitas, I. V. M. Silva e C. Barcellos contribuíram com a concepção do estudo, análise e interpretação dos dados, redação do artigo e aprovação da versão final. D. R. Xavier e E. L. Silva contribuíram com a análise e interpretação dos dados, redação do artigo e aprovação da versão final.

\section{Informações adicionais}

ORCID: Carlos Machado de Freitas (0000-00016626-9908); Isadora Vida de Mefano e Silva (00000001-9946-5147); Diego Ricardo Xavier (00000001-5259-7732); Eliane Lima e Silva (0000-00034608-5946); Christovam Barcellos (0000-00021161-2753).

\section{Agradecimentos}

Agradecemos as contribuições dos revisores, que tornaram o artigo mais direto e consistente.

\section{Referências}

1. World Health Organization. Sixty-fourth world health assembly. Geneva: World Health Organization; 2011.

2. World Health Organization. Fifty-eighth world health assembly. Health action in relation to crises and disasters, with particular emphasis on the earthquakes and tsunamis of 26 december 2004. Geneva: World Health Organization; 2005.

3. World Health Organization. Fifty-ninth world health assembly. Emergency preparedness and response. Geneva: World Health Organization; 2006.

4. World Health Organization. Sixty-first world health assembly. Geneva: World Health Organization; 2008.

5. World Meteorological Organization. Statement on the status of the global climate. Geneva: World Meteorological Organization; 2014.

6. Haines A, Ebi K. The imperative for climate action to protect health. N Engl J Med 2019; 380:263-73. 
7. Ministério da Saúde. Estratégia de saúde. In: Plano Nacional de Adaptação à Mudança do Clima. v. 2. Estratégias setoriais e temáticas : Portaria MMA no 150 de 10 de maio de 2016. Brasília: Ministério do Meio Ambiente; 2016. p. 190-212.

8. Freitas CM, Xavier DR, Sena ARM, Silva ES, Carvalho ML, Mazoto ML, et al. Desastres naturais e saúde: uma análise da situação do Brasil. Ciênc Saúde Colet 2014; 19:3645-56.

9. World Health Organization. Operational framework for building climate resilient health systems. Geneva: World Health Organization; 2015.

10. World Health Organization. Emergency risk management for health: overview. Geneva: World Health Organization; 2013.

11. Ministério da Integração Nacional. Instrução Normativa no 1, de 24 de agosto de 2012. Estabelece procedimentos e critérios para a decretação de situação de emergência ou estado de calamidade pública pelos Municípios, Estados e pelo Distrito Federal, e para o reconhecimento federal das situações de anormalidade decretadas pelos entes federativos e dá outras providências. Diário Oficial da União 2012; 30 ago.

12. Centro Universitário de Estudos e Pesquisas sobre Desastres, Universidade Federal de Santa Catarina. Atlas brasileiro de desastres naturais. Volume Brasil. 2a Ed. Florianópolis: Centro Universitário de Estudos e Pesquisas sobre Desastres, Universidade Federal de Santa Catarina; 2013.

13. Xavier DR, Barcellos CB, Barros HS, Magalhães MAFM, Matos VP, Pedroso MM. Organização, disponibilização e possibilidades de análise de dados sobre desastres de origem climática e seus impactos sobre a saúde no Brasil. Ciênc Saúde Colet 2014; 19:3657-68.

14. Kron W, Steuer M, Löw P, Wirtz A. How to deal properly with a natural catastrophe database: analysis of flood losses. Natural Hazards and Earth System Sciences 2012; 12:535-50.

15. Confederação Nacional de Municípios. Decretações de anormalidades causadas por desastres nos municípios brasileiros. Brasília: Confederação Nacional de Municípios; 2018.

16. Minervino AC, Duarte EC. Danos materiais causados à saúde pública e à sociedade decorrentes de inundações e enxurradas no Brasil, 2010-2014: dados originados dos sistemas de informação global e nacional. Ciênc Saúde Colet 2016; 21:685-94.

17. Organização Pan-Americana da Saúde; Ministério da Saúde. Desastres naturais e saúde no Brasil. Brasília: Organização Pan-Americana da Saúde/Ministério da Saúde; 2014.
18. Freitas CM, Carvalho ML, Ximenes EF, Arraes EF, Gomes JO. Vulnerabilidade socioambiental, redução de riscos de desastres e construção da resiliência: lições do terremoto no Haiti e das chuvas fortes na Região Serrana, Brasil. Ciênc Saúde Colet 2012; 17:1577-86.

19. Banco Mundial. Avaliação de perdas e danos. Inundações e deslizamentos na Região Serrana do Rio de Janeiro em 2011. Brasília: Banco Mundial; 2012.

20. Silva IVM. Vulnerabilidade institucional do setor saúde a desastres no Município de Nova Friburgo [Dissertação de Mestrado]. Rio de Janeiro: Escola Nacional de Saúde Pública Sergio Arouca, Fundação Oswaldo Cruz; 2019.

21. Rudowitz R, Rowland D, Shartzer A. Health care in New Orleans before and after hurricane Katrina. Health Aff (Millwood) 2006; 25:393-406.

22. Cuesta JG, Loenhout JAF, Lara-Banquesio ML, Isiderio JM, Aujoulat I, Guha-sapi D. The impact of typhoon Haiyan on health staff: a qualitative study in two hospitals in Eastern Visayas, the Philippines. Front Public Health 2018; 6:208.

23. Redlener IMD, Reilly MJ. Lessons from Sandy: preparing health systems for future disasters. N Engl J Med 2012; 367:2269-71.

24. Curtis S, Fair A, Aistow J, Val DV, Oven K. Impact of extreme weather events and climate change for health and social care systems. Environ Health 2017; 16 Suppl 1:128.

25. Carvalho ML. Reabilitação física e recuperação da saúde no contexto dos desastres naturais: estudo de caso em Nova Friburgo [Tese de Doutorado]. Rio de Janeiro: Escola Nacional de Saúde Pública Sergio Arouca, Fundação Oswaldo Cruz; 2017.

26. Kaiser Commission on Medical Aid and the Uninsured. Addressing the health care impact of hurricane Katrina. Washington DC: Kayser Family Foundation; 2005.

27. Miller AC, Arquilla B. Chronic diseases and natural hazards: impact of disasters on diabetic, renal, and cardiac patients. Prehosp Disaster Med 2008; 23:185-94.

28. Xavier DR, Barcellos C, Freitas CM. Eventos climáticos extremos e consequências sobre a saúde: o desastre de 2008 em Santa Catarina segundo diferentes fontes de informação. Ambiente \& Sociedade 2014; 17:273-94.

29. Instituto Brasileiro de Geografia e Estatística. Pesquisa de Informações Básicas Municipais. Rio de Janeiro: Instituto Brasileiro de Geografia e Estatística; 2013. 


\section{Abstract}

Natural disasters result in impacts on the population's health, damage to healthcare establishments, and, in extreme situations, the health systems' breakdown. National and global trends show an increase in the frequency of disasters associated with climate change. This article aims to analyze the impacts and economic costs of natural disasters for healthcare establishments, identifying the most frequent and costly types and distribution across the Brazilian territory, based on data recorded in Brazil's Integrated Disaster Information System (S2ID) from 2000 to 2015. A total of 15,950 records were systematized and analyzed, of which only 29.4\% of the events showed records of costs, totaling nearly BRL 4 billion. Climate disasters were the most frequent, but they did not account for the highest costs. In the cost per event ratio, the costs of hydrological disasters were 3.2 to 3.6 higher than for climate and geologic disasters. Pernambuco, Amazonas, and Santa Catarina were the states with highest total costs in millions of Brazilian reais. The North region, especially the state of Acre, had the highest cost per disaster. Despite the study's limitations (involving the records' quality), the data should be viewed as the tip of an iceberg, since the impacts go beyond the economic damages, impacting the infrastructure and resources that support services, compromising their capacity precisely when the population most needs health services.

Natural Disasters; Health Facilities; Costs and Cost Analysis; Climate Change

\section{Resumen}

Los desastres naturales provocan impactos en la salud de las poblaciones, perjuicios para establecimientos de salud y, en situaciones extremas, un colapso de los sistemas de salud. Tendencias nacionales y globales evidencian el aumento en la frecuencia de los desastres, asociados a los cambios climáticos. El objetivo de este artículo es analizar los impactos y costes económicos de los desastres naturales sobre los establecimientos de salud, identificando los tipos más frecuentes y de mayor coste, y su distribución en el territorio brasileño, teniendo como base los datos registrados en el Sistema Integrado de Información sobre Desastres (S2ID), durante el período de 2000 a 2015. Se sistematizaron y analizaron 15.950 registros, dentro de este universo solamente en un 29,4\% de las ocurrencias existían registros de costes, totalizando casi BRL 4 billones. Los desastres climatológicos fueron los más recurrentes, pero no los responsables de los costes más significativos. En la relación de costes por evento, los desastres hidrológicos tuvieron un coste 3,2 y 3,6 veces mayor que los meteorológicos y geológicos. Destacaron, en relación con su coste total en millones de reales, los estados de: Pernambuco, Amazonas y Santa Catarina. En lo que se refiere al coste por desastre en millones de reales resalta la Región Norte y en particular el estado de Acre. A pesar de las limitaciones de este estudio, relacionadas con la calidad de los registros, los datos presentados deben ser comprendidos como la punta visible de un iceberg, puesto que los impactos y perjuicios van más allá de la economía, afectando a la infraestructura y recursos que sirven de soporte para los servicios, comprometiendo la capacidad de oferta precisamente cuando la población necesita más los servicios de salud.

Desastres Naturales; Instituciones de Salud; Costos y Análisis de Costo; Cambio Climático
Recebido em 17/Jul/2019

Versão final reapresentada em 05/Dez/2019 Aprovado em 12/Dez/2019 